\title{
Measurement and calculation of the near field of a terahertz apertureless scanning optical microscope
}

\author{
Aurèle J. L. Adam, Nick C. J. van der Valk, and Paul C. M. Planken \\ Faculty of Applied Sciences, University of Technology Delft, Lorentzweg 1, 2628 CJ Delft, The Netherlands
}

Received September 20, 2006; revised December 13, 2006; accepted December 16, 2006; posted January 16, 2007 (Doc. ID 75197); published April 17, 2007

\begin{abstract}
We present measurements and calculations of the terahertz $(\mathrm{THz})$ electric field measured in the near field of a metal tip used in $\mathrm{THz}$ apertureless near-field optical microscopy (THz-ANSOM). An analytical model in which we treat the metal tip as a linear wire antenna allows us to predict almost all of the features observed in the measurements, such as the relatively slow decay of the near-field amplitude when the tip-crystal separation increases. When the tip-crystal separation is modulated, in conjunction with lock-in detection at the modulation frequency, a smaller $\mathrm{THz}$ spot size is observed underneath the tip. A comparison with analytical expressions shows that in this case the electric field originates predominantly from the tip apex, with negligible contributions from the tip shaft. In the unmodulated case, the observed signal is the spatial integral of the electrooptic (EO) effect over the interaction length between the $\mathrm{THz}$ near field and the probe laser pulse. In the modulated case, to a good approximation, we find that the signal is proportional to the value of the $\mathrm{THz}$ near field at the surface of the EO crystal only. (C) 2007 Optical Society of America

OCIS codes: $180.5810,300.670,110.3080,320.7160$.
\end{abstract}

\section{INTRODUCTION}

Terahertz apertureless near-field scanning optical microscopy (THz-ANSOM) is a new technique used to obtain a subwavelength resolution at terahertz $(\mathrm{THz})$ frequencies. In $\mathrm{THz}-\mathrm{ANSOM}, \mathrm{THz}$ pulses are incident on a metal tip having an apex of subwavelength dimensions, which is held close to a sample. One method to extract near-field information from the region of the tip apex is to measure the light that is scattered into the far field. This is typically done using electro-optic (EO) or photoconductive sampling techniques. ${ }^{1-3}$ The emission and detection patterns of $\mathrm{THz}$ pulses from such a metal-tip antenna have been studied experimentally by Walther et al. ${ }^{4}$ using a photoconductive switch. Another form of apertureless $\mathrm{THz}$ microscopy has recently been reported by Buersgens et al., ${ }^{5}$ who modulated the voltage between a tip and a conducting sample, while measuring the modulated $\mathrm{THz}$ signal in the far field.

In another method, the $\mathrm{THz}$ electric field is measured directly in the near field of the tip using EO sampling in a (100)-oriented GaP crystal. ${ }^{6}$ Both measurement methods have several important features in common: they measure the $\mathrm{THz}$ electric field instead of intensity and, because single-cycle broadband $\mathrm{THz}$ pulses are used, they measure in a large frequency bandwidth. Also, in both methods, a reduction of the $\mathrm{THz}$ near-field bandwidth compared with the bandwidth of the incident $\mathrm{THz}$ pulses is observed. This is caused by the antenna properties of the metal tips used in the experiments and was explained by Wang et al. ${ }^{7}$

Measuring the electric field directly in the near field of the tip has an important advantage: it gives direct and easy access to the properties of the near field. For ex- ample, only recently we obtained evidence that not only the tip apex, but also the tip shaft contributes significantly to the near field underneath the metal tip. ${ }^{8}$ Such information is essential to clarify and optimize the spatial resolution that can be obtained with these techniques and to help us understand the tip-sample interaction, which plays an important role in ANSOM experiments in general.

Here, we considerably expand our previous work on $\mathrm{THz}_{\mathrm{ANSOM}}{ }^{8}$ with additional measurements, calculations, and discussions on the nature of the near field of the metal tips. The $\mathrm{THz}$ electric field is measured directly in the near field of the metal tip, using an EO crystal. We find that when the tip-crystal distance is increased, the measured near-field signal initially decreases rapidly. However, when the tip-crystal distance increases to values much larger than the typical apex diameter, the observed near-field signal decreased surprisingly slowly. In contrast, when we modulate the tip-crystal distance using a piezo in conjunction with lock-in detection at the modulation frequency, we find that the measured $\mathrm{THz}$ nearfield signal decreases much more rapidly with the tipcrystal distance. A comparison with calculations shows that in the former case, the measured near field is dominated by contributions of the tip shaft, whereas in the latter case, it is dominated by contributions at the tip apex and corresponds to the value of the $\mathrm{THz}$ near field at the surface of the EO crystal. Theoretical models, which replace the tip by a sphere or a cone, fail to reproduce this behavior as they do not take into account the contributions to the near field from the tip shaft. In fact, we find that our results can only be understood by using the model of a linear wire antenna, which provides a correct 
description of the measured near field for a tip-crystal separation larger than $1 \mu \mathrm{m}$. Our results provide valuable insight into the nature of the near field of the metal tips used in ANSOM experiments in general, and in $\mathrm{THz}$ ANSOM experiments in particular.

This paper is organized as follows. In Section 2, we calculate first the near field of a sphere, followed by the near field of a wire antenna above a dielectric half-space, based on the quasi-static approximation. In Section 3, we show EO measurements of the $\mathrm{THz}$ electric field underneath the metal tip and also measurements of the near-field amplitude as a function of the tip-sample separation. The results are subsequently compared with the theoretical calculations. In Section 4, we discuss the validity and shortcomings of our model, and we compare our results with those obtained by others. Finally, in Section 5, a summary and conclusions are presented.

\section{THEORY}

\section{A. Introduction}

Important work has been done in the past concerning the near field of metal tips, also at visible frequencies. Finiteelement calculations, as well as finite-differential timedomain calculations have been published, ${ }^{9}$ although it is sometimes not easy to extract an intuitive physical picture from these numerical calculations. Analytical solutions for cones above dielectric half-planes have been developed, also for the optical domain, ${ }^{10}$ where plasmon resonances can play an important role.

An important consideration when calculating the near field around an object held above a dielectric half-plane, is the occurrence of the image-dipole effect, i.e., the generation of bound-surface charges and their effect on the electric field distribution around the metal object. We have chosen not to incorporate these effects in our models since for spheres and metal tips, they become important for tip-sphere-crystal separations smaller than the sphere or tip-apex diameter only and because they are difficult to calculate in general. As we will show in Subsection 2.B, this limits the validity of our main linear wire antenna model to tip-crystal separations larger than $1 \mu \mathrm{m}$. There is another reason why our model is limited to tip-crystal separations larger than $1 \mu \mathrm{m}$. Our linear wire antenna model does not include the finite thickness of the wire antenna. The metal tip is implicitly assumed to be infinitely thin, which, in theory, would give rise to an infinitely sharp apex and a corresponding infinitely high electric field, which is physically absurd. A minimum tip-crystal distance of $\sim 1 \mu \mathrm{m}$, a value that roughly corresponds to the experimental tip-apex diameter, avoids these problems. We note that numerical calculations typically also somehow have to deal with infinitely sharp fields near infinitely sharp objects. The tip-crystal separation limit mentioned here is thus by no means unique to our model.

The polarization of the dielectric is, however, taken into account to calculate the E field inside the dielectric. This essentially amounts to including the image dipole effects up to "zeroth" order. That is, we calculate the field inside the dielectric, without calculating the effect that the induced bound charges have on the field around the tip.
In our calculations, we assume that the quasi-static approximation is valid so that propagation effects, such as phase retardation, can be neglected. The derivation of the EO signal is done in several steps. First, the electric field of a point dipole pointing in the $z$ direction above a dielectric half-plane will be presented. Next, the results for the point dipole will be used to calculate the near field of an antenna above a dielectric half-plane, where we use the fact that the antenna can be thought of as consisting of infinitely many dipoles on a line. Then, the EO signal is calculated by integrating the electric field inside the crystal over the EO interaction distance. Finally, we obtain an expression for the EO signal for the case where we modulate the tip-sample distance. We would like to point out that an expression for the near-electric field of a wire antenna in free space is given by Balanis. ${ }^{11}$ Our derivation is somewhat different in that our starting point is the electric dipole. As we will see in Section 4, this allows us to present a physically intuitive picture to describe the results obtained when we modulate the tip-sample separation. However, to avoid duplications we will keep the derivation of the electric fields short and focus instead on the electro-optic signals.

\section{B. Field Created by the Dipole of a Sphere}

\section{Horizontal Dipole}

When we apply to a dielectric sphere an incident electric field, $E_{\text {incident }}$, the sphere becomes polarized with the dipole moment $\mathbf{p}_{\mathbf{d}}=\alpha \mathbf{E}_{\text {incident }}$, with $\alpha$ the polarizability of the sphere. The electric field outside a metal sphere is identical to the field of a point dipole located at the center of the sphere. Let us place this point dipole above a dielectric half-plane $\left(O_{x}, O_{y}\right)$, the EO crystal, at the point $A$ $\left(0,0, z_{o}\right)$ (see Fig. 1$)$. The electrostatic potential at a point $S(x, y, z)$ created by this electric point dipole can be written

$$
\begin{array}{ll}
V_{z>0}=\frac{\mathbf{p}_{\mathbf{d}} \cdot \mathbf{R}_{\mathbf{A S}}}{4 \pi \epsilon_{0}\left|R_{A S}\right|^{3}}+\frac{\epsilon-\epsilon_{0}}{\epsilon+\epsilon_{0}} \frac{\mathbf{p}_{\mathbf{d}} \cdot \mathbf{R}_{\mathbf{B S}}}{4 \pi \epsilon\left|R_{B S}\right|^{3}}, \quad(z>0), \\
V_{z \leqslant 0}=\frac{2 \epsilon}{\epsilon_{0}+\epsilon} \frac{\mathbf{p}_{\mathbf{d}} \cdot \mathbf{R}_{\mathbf{A S}}}{4 \pi \epsilon R_{A S}^{3}} & (z \leqslant 0),
\end{array}
$$

where $\mathbf{R}_{\mathrm{AS}}$ is the vector from $A$ to $S, R_{A S}$ is the distance $\left\|\mathbf{R}_{\mathrm{AS}}\right\|$ between the point $A$ and the point $S, \epsilon_{0}$ is the permittivity of the vacuum, and $\epsilon$ is the permittivity of the dielectric half-space. In our case, the incident electric field

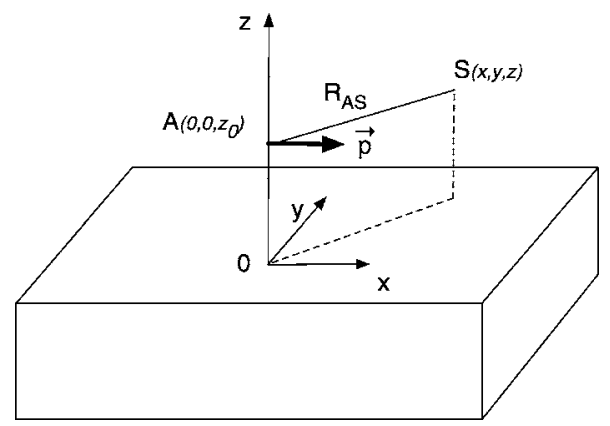

Fig. 1. Drawing of a vertical point dipole above a dielectric half-space. 
is parallel to the crystal and to the dipole moment. Then, vector $\mathbf{p}_{\mathbf{d}}$ points in the $x$ direction and the term $\mathbf{p}_{\mathbf{d}} \cdot \mathbf{R}_{\mathbf{A S}}$ is equal to $p_{d} x$. Our measurement method detects the $z$ component of the electric field inside the dielectric crystal for $z<0$. The potential at $z<0$ can thus be written

$$
V(S)=\frac{1}{4} \frac{2 \epsilon}{\epsilon_{0}+\epsilon} \frac{p_{d} x}{\pi \epsilon\left(x^{2}+y^{2}+\left(z-z_{0}\right)^{2}\right)^{3 / 2}} .
$$

The $z$ component of the electric field is equal to the partial derivative of the potential $V$ with respect to $z, E_{z}$ $=-\partial V / \partial z$, giving us

$$
E_{z}=\frac{3}{8} \frac{2 \epsilon}{\epsilon_{0}+\epsilon} \frac{p_{d} x\left(2 z-2 z_{0}\right)}{\pi \epsilon\left(x^{2}+y^{2}+\left(z-z_{0}\right)^{2}\right)^{5 / 2}} .
$$

Equation (3) immediately allows us to make a simple observation on the validity of the horizontal dipole as a model for a metal tip in ANSOM. When $x=0$ in Eq. (3), $E_{z}=0$, which contrasts with the experimental results obtained in Ref. 6 , that show a maximum for $E_{z}$ for $x=0$ and $y=0$.

We would like to point out again that Eq. (3) does not take into account the image dipole effect reported by Knoll. ${ }^{12}$ Knoll et al. have used a model of a sphere close to a dielectric half-space, in which the image dipole in turn changes the dipole moment of the sphere. This changes the field inside the crystal. The effect becomes only important for sphere-crystal separations smaller than the sphere diameter.

Another reason not to include this effect is that it assumes that the near field of the image dipole at the position of the "real" dipole is homogeneous across the sphere. This assumption is not correct and leads only to a qualitative understanding of the image dipole field enhancement.

\section{Vertical Dipole}

Let us assume that the dipole of the polarized sphere is not oriented horizontally, but vertically. We then obtain for the potential at $z<0$ :

$$
V(S)=\frac{1}{4} \frac{2 \epsilon}{\epsilon_{0}+\epsilon} \frac{p_{d}\left(z-z_{0}\right)}{\pi \epsilon\left(x^{2}+y^{2}+(z-z o)^{2}\right)^{3 / 2}} .
$$

The $z$ component of the electric field is then written

$$
\begin{aligned}
E_{z}= & -\frac{1}{4} \frac{2 \epsilon}{\epsilon_{0}+\epsilon} \frac{p_{d}}{\pi \epsilon\left(x^{2}+y^{2}+\left(z-z_{0}\right)^{2}\right)^{3 / 2}} \\
& +\frac{3}{8} \frac{(z-z o) p_{d}(2 z-2 z o)}{\pi \epsilon\left(x^{2}+y^{2}+(z-z o)^{2}\right)^{5 / 2}} .
\end{aligned}
$$

With $r^{2}=x^{2}+y^{2}$ and $\epsilon_{r}=\epsilon / \epsilon_{0}$, we can rewrite $E_{z}$ :

$$
\begin{aligned}
E_{z}= & -\frac{2}{\left(1+\epsilon_{r}\right)} \frac{p r^{2}}{4 \pi \epsilon_{0}\left(r^{2}+\left(z-z_{0}\right)^{2}\right)^{5 / 2}} \\
& +\frac{2}{\left(1+\epsilon_{r}\right)} \frac{2 p\left(z-z_{0}\right)^{2}}{4 \pi \epsilon_{0}\left(r^{2}+\left(z-z_{0}\right)^{2}\right)^{5 / 2}} .
\end{aligned}
$$

Along the $z$ axis at $x=y=0$, the field is then

$$
E_{z}(0,0, z)=\frac{1}{2} \frac{2 \epsilon}{\epsilon_{0}+\epsilon} \frac{p_{d}}{\pi \epsilon(z-z o)^{3}} .
$$

The field falls as $E_{z} \propto 1 /\left(z-z_{0}\right)^{3}$, not taking into account the image dipole effect.

In the experiments, it is the EO signal rather than the field itself that we detect. We assume that a laser pulse propagates through the near field of the metal tip in the $z$ direction in the crystal. The $z$ component of the field elliptically polarizes the probe pulse. Since we assume that we are operating in the quasi-static limit, the electric field distribution remains unchanged as the laser pulse propagates through the interaction volume. The EO signal is then directly proportional to

$$
S_{E O} \propto \int_{-p}^{-q} E_{z} \mathrm{~d} z,
$$

where $p$ and $q$ define the beginning and the end position of the EO-interaction length. The integral is easily evaluated and yields

$$
S_{E O} \propto \frac{p_{d}}{4 \pi \epsilon_{0}}\left(\frac{1}{\left(q+z_{0}\right)^{2}}-\frac{1}{\left(p+z_{0}\right)^{2}}\right),
$$

where we have assumed $x=y=0$, since we are only interested at this point in the field directly underneath the dipole.

In Fig. 2, we plot the calculated EO signal as a function of the dipole-crystal separation for several integration path lengths. In this calculation, we assume that $q=0$, that is, integration up to the crystal surface. One can see that this model leads to a fast decrease of the signal when the dipole-crystal separation increases from 1 to $\sim 5 \mu \mathrm{m}$. The figure also shows that on a linear scale, changing the integration path length has only a small effect on the calculated EO signal.

\section{Field of a Wire Antenna}

\section{Vertical Wire Antenna}

We can use the expression for the point dipole, Eq. (6), to calculate the $z$ component of a thin antenna above a di-

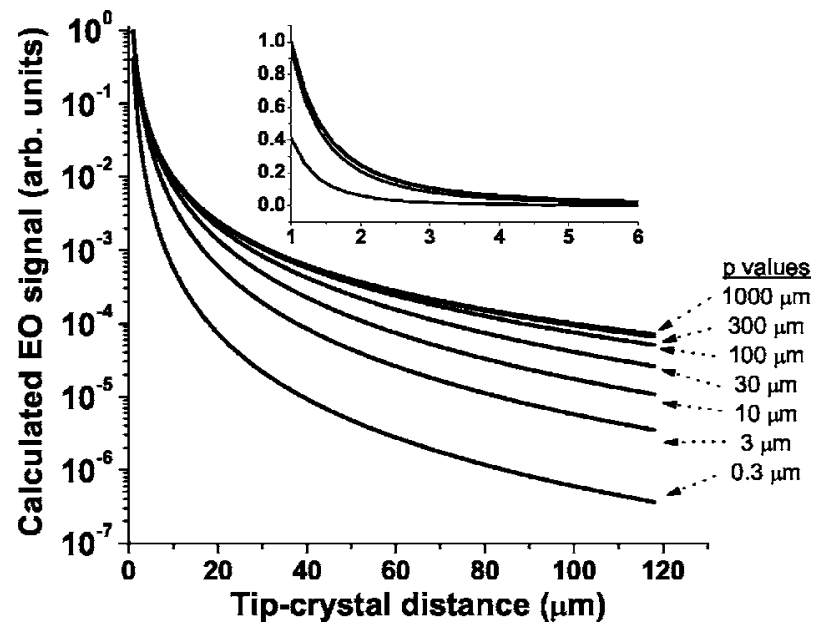

Fig. 2. EO signal calculated for the sphere model at different lengths of integration $p$ for $q=0$. Note that the curves are not normalized. The inset is a close up with a linear scale. 


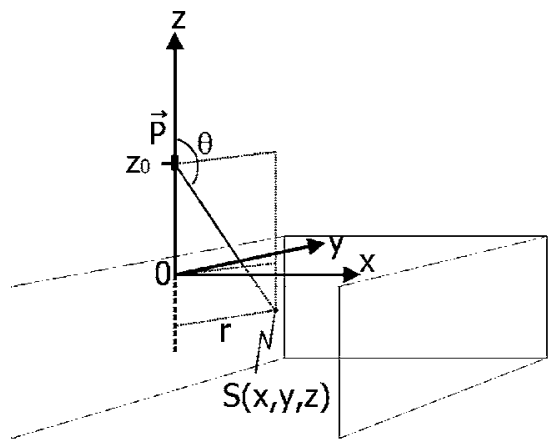

(a)

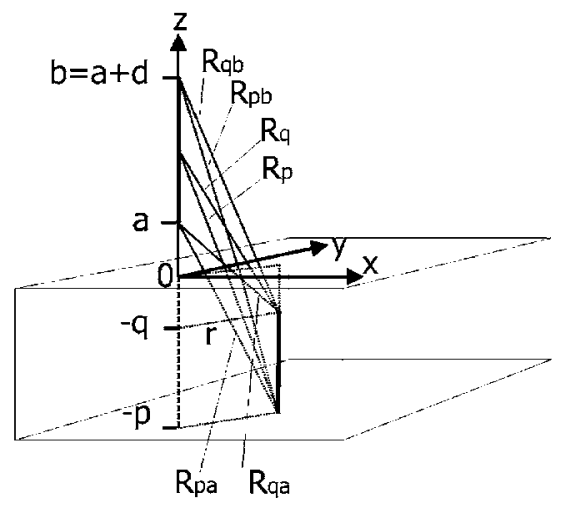

(c)

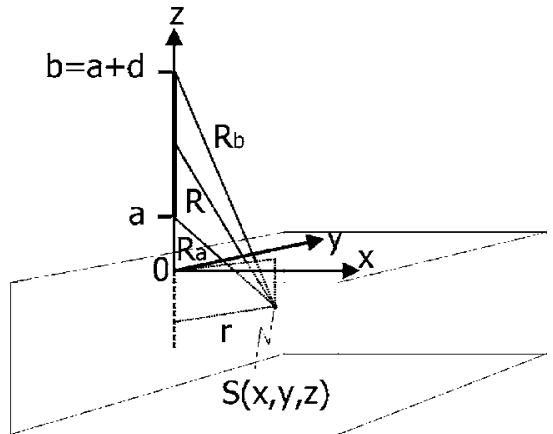

(b)

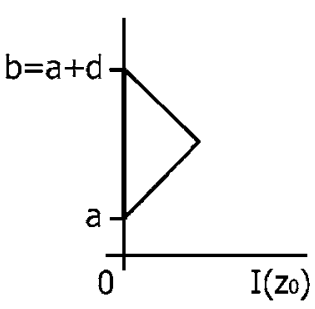

(d)

Fig. 3. (a) Drawing of a vertical point dipole above a dielectric half-space. (b) Vertical wire antenna above a dielectric half-space. (c) Vertical wire antenna above a dielectric half-space showing the path inside the crystal along which the EO effect is integrated. (d) Schematic of the vertical wire antenna with a triangular current distribution.

electric half-space. The wire antenna then consists of many point dipoles on a line with a strength, which depends on the position on the line. We consider a short segment $\mathrm{d} z_{0}$ at $z_{0}$ of the antenna and assume that this antenna segment has a dipole $p\left(z_{0}, t\right)$ [Fig. 3(b)]. We assume that the dipole is slowly varying (or alternatively, that the antenna is short) so that phase-retardation effects can be neglected and all dipoles along the wire oscillate in phase. The current in the wire at position $z_{0}$, which gives rise to the dipole, can then be written as $I\left(z_{0}, t\right)=I_{1}\left(z_{0}\right) I_{2}(t)$, where the time-dependent part $I_{2}(t)$ is the same for all the dipoles in the wire. The current is related to the dipole $p$ according to

$$
\begin{aligned}
p\left(z_{0}, t\right) & =\mathrm{d} z_{0} \int_{-\infty}^{t} I\left(z_{0}, t^{\prime}\right) \mathrm{d} t^{\prime}=\int_{-\infty}^{t} I_{2}\left(t^{\prime}\right) \mathrm{d} t^{\prime} I_{1}\left(z_{0}\right) \mathrm{d} z_{0} \\
& \equiv G(t) I_{1}\left(z_{0}\right) \mathrm{d} z_{0} .
\end{aligned}
$$

All the time dependence of the dipole is now contained by the term $G(t)$. Using Eq. (6), we can thus write for the $z$ component of the electric field $\mathrm{d} E_{z}$ of the antenna section $\mathrm{d} z_{0}$ in the dielectric half-space $(z \leqslant 0)$ :

$$
\begin{aligned}
\mathrm{d} E_{z}= & -\frac{2}{\left(1+\epsilon_{r}\right)} \frac{G(t) I_{1}\left(z_{0}\right) r^{2} \mathrm{~d} z_{0}}{4 \pi \epsilon_{0}\left(r^{2}+\left(z-z_{0}\right)^{2}\right)^{5 / 2}} \\
& +\frac{2}{\left(1+\epsilon_{r}\right)} \frac{2 G(t) I_{1}\left(z_{0}\right)\left(z-z_{0}\right)^{2} \mathrm{~d} z_{0}}{4 \pi \epsilon_{0}\left(r^{2}+\left(z-z_{0}\right)^{2}\right)^{5 / 2}} .
\end{aligned}
$$

To calculate the $z$ component of the electric field of the complete antenna, we have to integrate Eq. (11) over the entire length of the antenna, from $z_{0}=a$ to $z_{0}=b$ :

$$
\begin{aligned}
E_{z}= & -\frac{2 G(t)}{4 \pi \epsilon_{0}\left(1+\epsilon_{r}\right)} \int_{a}^{b}\left(\frac{I_{1}\left(z_{0}\right) r^{2}}{\left(r^{2}+\left(z-z_{0}\right)^{2}\right)^{5 / 2}}\right. \\
& \left.-\frac{2 I_{1}\left(z_{0}\right)\left(z-z_{0}\right)^{2}}{\left(r^{2}+\left(z-z_{0}\right)^{2}\right)^{5 / 2}}\right) \mathrm{d} z_{0} .
\end{aligned}
$$

At this point, the current is not specified and thus, the integral cannot be evaluated. For obvious reasons, the current has to be zero at both ends of the antenna. A rectangular distribution is then not realistic. Let us assume then that the current distribution in the wire is triangular ${ }^{11}$ along the antenna as shown in Fig. 3(d), with a maximum in the middle. This current distribution is given by

$$
\begin{aligned}
& I_{1}\left(z_{0}\right)=C_{0}\left(z_{0}-a\right) \quad\left(a<z_{0}<\left(a+\frac{b-a}{2}\right)\right), \\
& I_{1}\left(z_{0}\right)=C_{0}\left(b-z_{0}\right) \quad\left(\left(a+\frac{b-a}{2}\right)<z_{0}<b\right),
\end{aligned}
$$

with $C_{0}$ a constant proportional to the current amplitude. We can then rewrite Eq. (12) as 


$$
\begin{aligned}
E_{z}= & -\frac{2 C_{0} G(t)}{4 \pi \epsilon_{0}\left(1+\epsilon_{r}\right)} \int_{a}^{(a+b) / 2}\left(\frac{\left(z_{0}-a\right) r^{2}}{\left(r^{2}+\left(z-z_{0}\right)^{2}\right)^{5 / 2}}\right. \\
& \left.-\frac{2\left(z_{0}-a\right)\left(z-z_{0}\right)^{2}}{\left(r^{2}+\left(z-z_{0}\right)^{2}\right)^{5 / 2}}\right) \mathrm{d} z_{0} \\
& -\frac{2 C_{0} G(t)}{4 \pi \epsilon_{0}\left(1+\epsilon_{r}\right)} \int_{(a+b) / 2}^{b}\left(\frac{\left(b-z_{0}\right) r^{2}}{\left(r^{2}+\left(z-z_{0}\right)^{2}\right)^{5 / 2}}\right. \\
& \left.-\frac{2\left(b-z_{0}\right)\left(z-z_{0}\right)^{2}}{\left(r^{2}+\left(z-z_{0}\right)^{2}\right)^{5 / 2}}\right) \mathrm{d} z_{0} .
\end{aligned}
$$

We can easily evaluate these integrals by defining

$$
\begin{aligned}
& R^{2}=r^{2}+\left(z-\frac{a+b}{2}\right)^{2}, \\
& R_{a}^{2}=r^{2}+(z-a)^{2}, \\
& R_{b}^{2}=r^{2}+(z-b)^{2},
\end{aligned}
$$

where $R, R_{a}$, and $R_{b}$ have the geometrical interpretation shown in Fig. 3(b). We then obtain

$$
E_{z}=\frac{2 C_{0} G(t)}{4 \pi \epsilon_{0}\left(1+\epsilon_{r}\right)}\left(\frac{1}{R_{a}}+\frac{1}{R_{b}}-\frac{2}{R}\right) \quad(z \leqslant 0)
$$

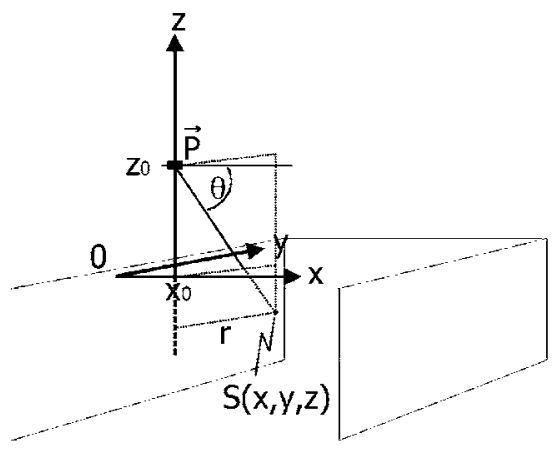

(a)

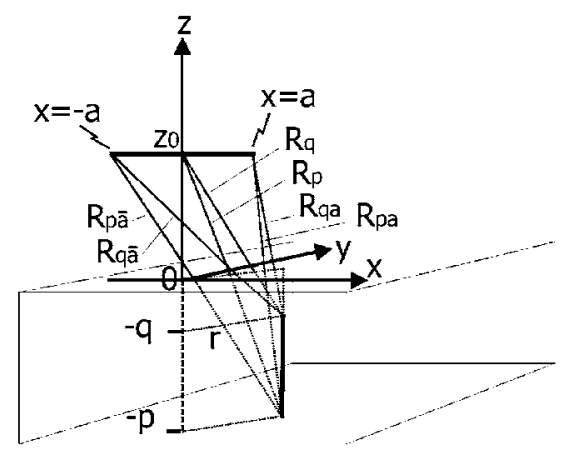

(c)
2. Horizontal Wire Antenna

The electric field at $(x, y, z), z \leqslant 0$, of a horizontal dipole oriented in the $x$ direction positioned at $\left(x_{0}, 0, z_{0}\right)$ above a dielectric half-space is given by Eq. (3):

$$
E_{z}=\frac{6 p\left(x-x_{0}\right)\left(z-z_{0}\right)}{\left(1+\epsilon_{r}\right) 4 \pi \epsilon_{0}\left(\left(x-x_{0}\right)^{2}+y^{2}+\left(z-z_{0}\right)^{2}\right)^{5 / 2}} .
$$

For a horizontal wire antenna of length $2 a$ oriented along the $x$ axis from $x=-a$ to $x=a$, at height $z_{0}$ above the dielectric half-space, the electric field in the dielectric at position $(x, y, z)$ is given by

$$
\begin{array}{r}
E_{z}=-\frac{2 C_{0} G(t)}{4 \epsilon_{0} \pi\left(1+\epsilon_{r}\right)} \frac{\left(z-z_{0}\right)}{\left(\left(z-z_{0}\right)^{2}+y^{2}\right)}\left(\frac{x+a}{R_{\bar{a}}}+\frac{x-a}{R_{a}}-\frac{2 x}{R}\right) \\
(z \leqslant 0),
\end{array}
$$

where $R, R_{a}$, and $R_{\bar{\alpha}}$ have the geometrical interpretation indicated in Fig. 4(b) and are defined by

$$
\begin{aligned}
& R^{2}=x^{2}+y^{2}+\left(z-z_{0}\right)^{2}, \\
& R_{a}^{2}=(x-a)^{2}+y^{2}+\left(z-z_{0}\right)^{2}, \\
& R_{\bar{a}}^{2}=(x+a)^{2}+y^{2}+\left(z-z_{0}\right)^{2} .
\end{aligned}
$$

We have also assumed a triangular current distribution, see Fig. 4(d), similar to that for the vertical wire antenna.

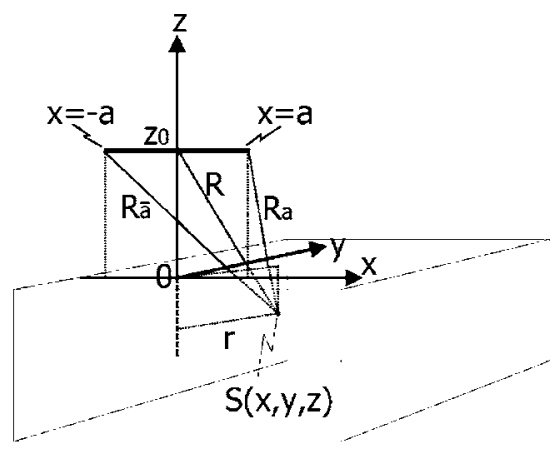

(b)

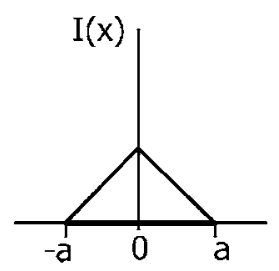

(d)

Fig. 4. (a) Drawing of a horizontal point dipole above a dielectric half-space. (b) Horizontal wire antenna above a dielectric half-space. (c) Horizontal wire antenna above a dielectric half-space showing the path inside the crystal along which the EO effect is integrated. (d) Schematic of the horizontal wire antenna with a triangular current distribution. 


\section{Electro-Optic Signal}

1. Vertical Wire Antenna

To compare the calculations with the measurements, as described in the next section, we now calculate the EOdetection signal. Referring to Fig. 3(c), we assume that a laser pulse propagates through the near field of the metal tip in the $z$ direction. The $z$ component of the field elliptically polarizes the probe pulse. Since we are operating in the quasi-static limit, we can assume that the electric field distribution remains unchanged as the laser pulse propagates through the interaction volume. The EO signal is then directly proportional to

$$
S_{E O} \propto \int_{-p}^{-q} E_{z} \mathrm{~d} z,
$$

where $p$ and $q$ define the EO-interaction length inside the crystal. The laser pulse is assumed to propagate in the $z$ direction and to be infinitely short. The integral is easily evaluated and yields

$$
\begin{aligned}
S_{E O} \propto & \frac{2 C_{0} G(t)}{4 \pi \epsilon_{0}\left(1+\epsilon_{r}\right)}\left[\ln \left(\frac{-q-a+R_{q a}}{-p-a+R_{p a}}\right)+\ln \left(\frac{-q-b+R_{p b}}{-p-b+R_{p b}}\right)\right. \\
& \left.-2 \ln \left(\frac{-b-a-2 q+2 R_{q}}{-b-a-2 p+2 R_{p}}\right)\right] .
\end{aligned}
$$

We have used the following definitions of the $R \mathrm{~s}$, which have the geometrical interpretation indicated in Fig. 3(c).

$$
\begin{aligned}
& R_{p a}^{2}=r^{2}+(a+p)^{2}, \\
& R_{p b}^{2}=r^{2}+(b+p)^{2}, \\
& R_{q a}^{2}=r^{2}+(a+q)^{2}, \\
& R_{q b}^{2}=r^{2}+(b+q)^{2}, \\
& R_{p}^{2}=r^{2}+\left(p+\frac{a+b}{2}\right)^{2}, \\
& R_{q}^{2}=r^{2}+\left(q+\frac{a+b}{2}\right)^{2} .
\end{aligned}
$$

\section{Horizontal Wire Antenna}

The EO signal for the horizontal antenna is given by

$$
\begin{aligned}
S_{E O} \propto & \frac{C_{0} G(t)}{4 \pi \epsilon_{0}\left(1+\epsilon_{r}\right)} \times\left[\ln \left(\frac{\left[\left(p+z_{0}\right)^{2}+y^{2}\right]\left[\left((x-a)^{2}+y^{2}+(x-a) R_{q a}\right)^{2}+y^{2}\left(q+z_{0}\right)^{2}\right]}{\left.\left[\left(q+z_{0}\right)^{2}+y^{2}\right]\left[(x-a)^{2}+y^{2}+(x-a) R_{p a}\right)^{2}+y^{2}\left(q+z_{0}\right)^{2}\right]}\right)\right. \\
& \left.+\ln \left(\frac{\left[\left(p+z_{0}\right)^{2}+y^{2}\right]\left[\left((x+a)^{2}+y^{2}+(x+a) R_{q \bar{a}}\right)^{2}+y^{2}\left(q+z_{0}\right)^{2}\right]}{\left[\left(q+z_{0}\right)^{2}+y^{2}\right]\left[\left((x+a)^{2}+y^{2}+(x+a) R_{p \bar{a}}\right)^{2}+y^{2}\left(q+z_{0}\right)^{2}\right]}\right)-2 \ln \left(\frac{\left[\left(p+z_{0}\right)^{2}+y^{2}\right]\left[\left(x^{2}+y^{2}+x R_{q}\right)^{2}+y^{2}\left(q+z_{0}\right)^{2}\right]}{\left[\left(q+z_{0}\right)^{2}+y^{2}\right]\left[\left(x^{2}+y^{2}+x R_{p}\right)^{2}+y^{2}\left(q+z_{0}\right)^{2}\right]}\right)\right],
\end{aligned}
$$

where the $R$ s are defined by

$$
\begin{aligned}
& R_{p a}^{2}=(x-a)^{2}+y^{2}+\left(p+z_{0}\right)^{2}, \\
& R_{p \bar{a}}^{2}=(x+a)^{2}+y^{2}+\left(p+z_{0}\right)^{2}, \\
& R_{q a}^{2}=(x-a)^{2}+y^{2}+\left(q+z_{0}\right)^{2}, \\
& R_{q \bar{a}}^{2}=(x+a)^{2}+y^{2}+\left(q+z_{0}\right)^{2}, \\
& R_{p}^{2}=x^{2}+y^{2}+\left(p+z_{0}\right)^{2}, \\
& R_{q}^{2}=x^{2}+y^{2}+\left(q+z_{0}\right)^{2} .
\end{aligned}
$$

The geometrical interpretation of the $R \mathrm{~s}$ is given in Fig. $4(\mathrm{c})$.

\section{E. Electro-Optic Signal Under the Tip}

\section{Vertical Wire Antenna}

In many of the experiments, the EO signal is measured directly underneath the metal tip, where $r=0$. To evaluate
Eq. (21) at $r=0$, let us take a look at the first term within square brackets. This term can be written more explicitly as

$$
\ln \left(\frac{-q-a+\left(r^{2}+(a+q)^{2}\right)^{1 / 2}}{-p-a+\left(r^{2}+(a+p)^{2}\right)^{1 / 2}}\right) .
$$

To evaluate this term, we develop the square-root terms into a series around $r=0$ :

$$
\begin{aligned}
& \left(r^{2}+(a+q)^{2}\right)^{1 / 2} \simeq(a+q)+\frac{r^{2}}{2(a+q)}-\frac{r^{4}}{8(a+q)^{3}}+O\left(r^{6}\right), \\
& \left(r^{2}+(a+p)^{2}\right)^{1 / 2} \simeq(a+p)+\frac{r^{2}}{2(a+p)}-\frac{r^{4}}{8(a+p)^{3}}+O\left(r^{6}\right) .
\end{aligned}
$$

Inserting this into Eq. (25) and taking the limit of $r$ $\rightarrow 0$ gives

$$
\ln \left(\frac{a+p}{a+q}\right)
$$


We can do the same for the other terms in square brackets of Eq. (21), so that we can write for the signal measured directly underneath the antenna:

$$
\begin{aligned}
S_{E O} \propto \frac{2 C_{0} G(t)}{4 \pi \epsilon_{0}\left(1+\epsilon_{r}\right)}\left[\ln \left(\frac{a+p}{a+q}\right)+\ln \left(\frac{b+p}{b+q}\right)\right. \\
\left.-2 \ln \left(\frac{\frac{a+b}{2}+p}{\frac{a+b}{2}+q}\right)\right] \quad(r=0) .
\end{aligned}
$$

This answer is surprising in at least one respect. The occurrence of the natural logarithm in Eq. (28) indicates that when the tip-sample distance is increased, the measured EO signal decays only slowly. We note that Eq. (28) can be further simplified in the purely theoretical limit of an extremely long antenna $(b \rightarrow \infty)$. We find

$$
S_{E O} \propto \frac{2 C_{0} G(t)}{4 \pi \epsilon_{0}\left(1+\epsilon_{r}\right)} \ln \left(\frac{a+p}{a+q}\right) .
$$

It should be noted that for a given wavelength, taking the limit of $b$ to infinity means that we eventually reach a situation where the formulas are no longer valid: the quasi-static approximation implicitly assumes that the antenna is shorter than the wavelength, so that propagation effects (phase retardation) can be neglected.

\section{Horizontal Wire Antenna}

Directly underneath a horizontal wire antenna at $x=y$ $=0$, in fact, in the entire $x=0$ plane, the electric field is purely horizontal. There is therefore no $z$ component and thus no EO signal in our (110)-oriented detection crystal.

\section{F. Cone}

Since the tip apex resembles a cone, approximating the tip by a cone seems reasonable. This was done in the past by Bladel $^{13}$ and Cory. ${ }^{14}$ For our thin wire antennas, we can assume that the cone angle approaches 0 . In that case, the field in the neighborhood of the cone can be written as

$$
\mathbf{E} \propto \frac{1}{R} \cos \left(\frac{\theta}{2}\right) \mathbf{u}_{\mathbf{r}}-\frac{1}{R} \sin \left(\frac{\theta}{2}\right) \mathbf{u}_{\theta},
$$

with $R$ being the distance from the end of the apex and $\theta$ being the angle with the axis of the tip. $\mathbf{u}_{\mathbf{R}}$ and $\mathbf{u}_{\theta}$ are the vectors in the spherical coordinate system. The electric field directly under the tip where $\theta=0$, is then given by

$$
\mathbf{E} \propto \frac{1}{R} \hat{\mathbf{z}},
$$

and thus has a component only in the $z$ direction. Surprisingly, the field decreases as $1 / R$. For small cone angles, then, the field calculated directly underneath the tip is identical to the field directly underneath a linear wire antenna [Eq. (16)], where there we assume a long antenna such that $R, R_{b} \ll R_{a}$.

\section{G. Tip-Sample Modulation}

In some experiments we modulate the tip-sample separation in conjunction with lock-in detection at the modulation frequency. Lock-in detection ensures that only the component of the $\mathrm{THz}$ near field that is affected by the modulation is observed. What is measured therefore, for small enough modulation amplitudes, is proportional to the first spatial derivative of the EO signal $S_{E O}$ with respect to spatial modulation coordinate.

\section{Vertical Wire Antenna}

In the experiments, the antenna height $a$ above the crystal is modulated at position $a_{0}$. This automatically means that $b$ is modulated too and we therefore write $b=a_{0}+d$, where $d$ is the length of the antenna. The first derivative of the EO signal, Eq. (21), evaluated at $a=a_{0}$ is

$$
\begin{aligned}
S_{E O}^{\prime} \equiv & {\left[\frac{\partial S_{E O}}{\partial a}\right]_{a=a_{0}} \propto \frac{2 C_{0} G(t)}{4 \pi \epsilon_{0}\left(1+\epsilon_{r}\right)}\left(\frac{1}{R_{p b}}+\frac{1}{R_{p a_{0}}}-\frac{1}{R_{q a_{0}}}-\frac{1}{R_{q b}}\right.} \\
& \left.+\frac{2}{R_{q}}-\frac{2}{R_{p}}\right)
\end{aligned}
$$

where the $R$ s are defined in Eq. (22), with $a$ replaced everywhere with $a_{0}$ and with $b=a_{0}+d$. The modulated EO signal, Eq. (32), shows some resemblance to Eq. (16), which represents the (near) field inside the crystal. The resemblance becomes even more striking when we assume that $p$ is large and $q$ is small (integration over the whole EO crystal), so that the three terms in Eq. (32) containing $p$ can be neglected. In this case, the measured signal can be approximated by

$$
S_{E O}^{\prime} \propto-\frac{2 C_{0} G(t)}{4 \pi \epsilon_{0}\left(1+\epsilon_{r}\right)}\left(\frac{1}{R_{q a_{0}}}+\frac{1}{R_{q b}}-\frac{2}{R_{q}}\right) .
$$

For $q=0$ (integration all the way to the surface) this is identical to Eq. (16), when in that equation we take the value of the electric field at the surface $(z=0)$. We thus find that in the approximation $p$ is large and $q=0$, the EO signal measured when the tip-sample distance is modulated, has exactly the same functional dependence on distance, as the expression of the electric field of a vertical wire antenna at $z=0$. A simple hand waiving explanation for why this is so, is that EO detection corresponds to spatial integration of the electric field in the $z$ direction, whereas tip-sample distance modulation in the $z$ direction, with subsequent detection at the modulation frequency, corresponds to differentiation in the $z$ direction. Their combined action thus leaves the original equation almost unchanged.

\section{Horizontal Wire Antenna}

In a calculation very similar to that for the vertical wire antenna, we can derive the measured EO signal induced by a horizontal wire antenna when the antenna-sample distance is modulated, with subsequent lock-in detection at the modulation frequency. For simplicity, only the signal in the $z x$ plane will be calculated, allowing us to put $y=0$. The first derivative of the EO signal evaluated at $z_{0}=z_{1}$ is 


$$
\begin{aligned}
S_{E O}^{\prime} \equiv & {\left[\frac{\partial S_{E O}}{\partial z_{0}}\right]_{z_{0}=z_{1}} \propto \frac{C_{0} G(t)}{4 \pi \epsilon_{0}\left(1+\epsilon_{r}\right)}\left(\frac{q+z_{1}}{R_{q a}\left(x-a+R_{q a}\right)}\right.} \\
& -\frac{p+z_{1}}{R_{p a}\left(x-a+R_{p a}\right)}+\frac{q+z_{1}}{R_{q \bar{a}}\left(x+a+R_{q \bar{a}}\right)} \\
& \left.-\frac{p+z_{1}}{R_{p \bar{a}}\left(x+a+R_{p \bar{a}}\right)}-\frac{2\left(q+z_{1}\right)}{R_{q}\left(x+R_{q}\right)}+\frac{2\left(p+z_{1}\right)}{R_{p}\left(x+R_{p}\right)}\right),
\end{aligned}
$$

where the $R$ s are defined as in Eq. (24), but with $z$ replaced with $z_{1}$ and $y=0$. This equation can be further simplified if we assume that $p$ is large and that $q$ is small, so that the terms in Eq. (34) that include $p$ can be neglected. After slightly rewriting the remaining terms, we obtain

$$
S_{E O}^{\prime} \propto-\frac{C_{0} G(t)}{4 \pi \epsilon_{0}\left(1+\epsilon_{r}\right)} \frac{1}{\left(q+z_{1}\right)}\left(\frac{x-a}{R_{q a}}+\frac{x+a}{R_{q \bar{a}}}-\frac{2 x}{R_{q}}\right),
$$

which for $q=0$ (integration all the way to the crystal surface) has the same functional dependence as the electric field of a horizontal wire antenna give by Eq. (18), when in that equation we take $y=0$, use $z_{0}=z_{1}$ for the antenna height above the crystal, and calculate the electric field at the crystal surface at $z=0$. To a good approximation therefore, when we modulate the separation between a horizontal tip and the EO crystal, the measured EO signal (for $y=0$ ) represents the electric field measured at the surface of the crystal.

\section{RESULTS}

\section{A. Setup}

The setup used for the measurements has been described in more detail elsewhere. ${ }^{6}$ A metal tip with an apex of subwavelength dimensions is held close to the surface of a GaP EO crystal. The tip is placed nearly perpendicular to the surface of the crystal with a slight tilt (few degrees) to ensure that a component of the incident field is oriented parallel to the long axis of the tip. Such a small tilt has practically no effect on the results predicted by our model. The tip radius is on the order of $1 \mu \mathrm{m}$ and the tip cone angle is equal to a few degrees. For distances away from the tip, larger than $\sim 1 \mu \mathrm{m}$, therefore, the antenna can be approximated by an infinitely thin wire antenna.

The tip is illuminated with $\mathrm{THz}$ pulses generated in a photoconductive switch. ${ }^{15}$ The $\mathrm{THz}$ pulses are polarized parallel to the crystal surface with a component along the length of the tip shaft. From the back of the crystal, a probe laser pulse is tightly focused to a $4 \mu \mathrm{m}$ spot size underneath the metal tip. Due to the EO effect in the GaP crystal, the $\mathrm{THz}$ field elliptically polarizes the probe beam. The degree of ellipticity is measured in a standard differential detector setup and is linearly proportional to the $\mathrm{THz}$ electric field. The GaP crystal has the [001] orientation in order to detect only the $\mathrm{THz}$ field components polarized perpendicularly to the crystal surface.

To establish the validity of the model described earlier, we have performed two types of EO measurements of the $\mathrm{THz}$ electric fields near the tip: one with the tip nonvi- brating and one with the tip attached to a piezolinear actuator which is oscillating vertically. In both cases, the EO signal as a function of the tip-crystal distance is measured.

\section{B. Nonvibrating Tip}

The tip is placed in the vicinity of the crystal but not in contact. Using micrometers, we manually move the position of the tip in the $x y$ plane parallel to the crystal surface until a maximum in the signal is obtained. The position where the signal is maximal corresponds to a tip position directly above the focused probe beam. This is visually checked using a visible microscope in conjunction with a CDD camera. The incoming $\mathrm{THz}$ beam has a diameter much larger than the tip apex, so we can assume that the $\mathrm{THz}$ pulse illuminates the tip uniformly. We repeated the measurement with several tips on different crystal locations to check for reproducibility of the results. The azimuthal orientation of the crystal is also important. When rotating the crystal in the $x y$ plane, the signal changes. The crystal azimuthal angle is rotated until a maximum in the EO signal is obtained.

Figure 5 shows, in dotted curves, the measured peak intensity as a function of the tip-crystal separation $a$. Note that the unit along the horizontal axis is in micrometers and not in fractions of a wavelength. There are two reasons for this. First, even though Maxwell's equations are scalable, suggesting that our model may be applicable to a far larger wavelength range, our results are derived in the quasi-static approximation where the frequency does not play a role. Second, our $\mathrm{THz}$ source is broadband and so we would have to arbitrarily pick one reference wavelength out of the multitude of wavelengths present in the pulse spectrum. The measurement covers a total distance of $120 \mu \mathrm{m}$. We have chosen the $0 \mu \mathrm{m}$ position arbitrarily as the position where the signal reached is maximum. This corresponds to a position where the tipcrystal separation is less than $1 \mu \mathrm{m}$. When removing the tip from the crystal, the signal rapidly decreases to half of

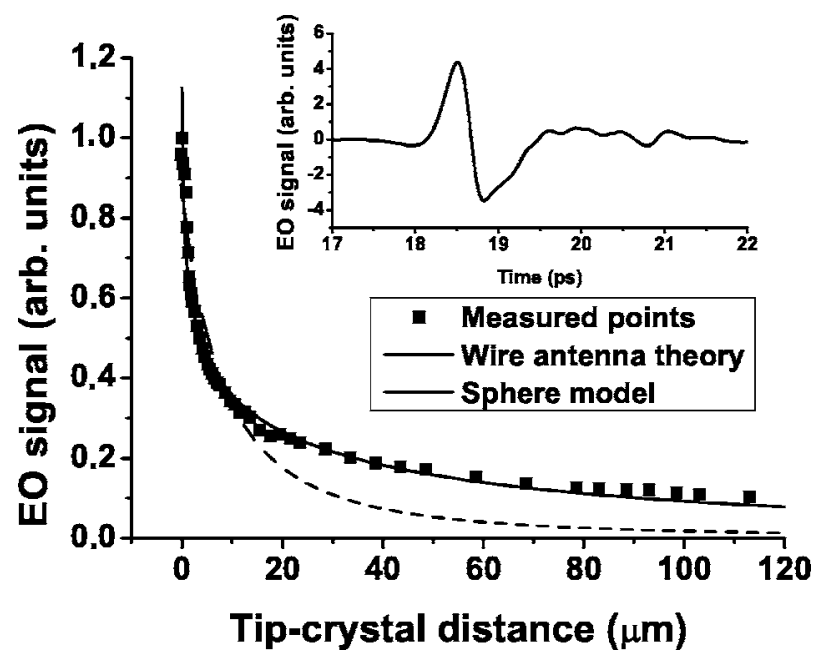

Fig. 5. Measured EO signal (squares) as a function of the tipcrystal distance. The solid curve is calculated using the antenna model and the dotted curve is calculated using the sphere model. The inset is a signal recorded by the differential detector as a function of time for a tip-crystal distance of $1 \mu \mathrm{m}$. 
its maximum value in less than $5 \mu \mathrm{m}$. This clearly indicates near-field behavior. Further away, the signal still decreases but at a moderate rate. At $120 \mu \mathrm{m}$ from the crystal, a signal can still be measured, at $10 \%$ of the maximum value.

In the same figure, we plotted the results of the models described above: the solid curve represents the simple sphere model, the dotted one represents the antenna model. Parameters $p, q$, and $a$ have been adjusted manually in order to obtain the best fit with the measured curve. The figure clearly shows that for tip-crystal separations larger than $10 \mu \mathrm{m}$, the sphere model clearly fails to reproduce the measurement as it underestimated the strength of the EO signal. The wire antenna model, however, reproduces the measurements on the whole $120 \mu \mathrm{m}$ range and is thus shown to be a much better model to describe our measurement than the sphere model.

The values of the parameters obtained in fitting the vertical wire antenna model to the experimental data have a real physical meaning: the distance of integration inside the substrate is $\sim 150 \mu \mathrm{m}$ and $d$, the length of the tip, is $3.5 \mathrm{~mm}$. It should be noted that the quasi-static approximation used in our model is not valid anymore for an antenna with a length of $3.5 \mathrm{~mm}$, which is three times longer than the wavelength. This value was obtained after fine-tuning the model parameters to fit the data and should be considered an order of magnitude estimate only. In addition, we find that the contribution of the most distant part of the tip to the field underneath the tip is negligible compared with the contributions from the lower parts of the tip shaft anyway, making the model insensitive to changes in the tip length for long tips.

\section{Vibrating Tip}

Recently, we showed that when the tip-crystal distance is modulated, in conjunction with lock-in detection at the modulation frequency, the measured intensity distribution underneath the metal tip originates from the tip apex alone. ${ }^{8}$ Without modulation, the signal is dominated by contributions from the tip shaft, which completely overwhelm the contributions from the apex. We now wish to check if the functional dependence on the distance of the EO signal when the tip-crystal distance is modulated can be described by our model.

The EO signal modulated by a vibrating tip is expected to be directly proportional to the $\mathrm{THz}$ field according to Eq. (33). To test this, the tip is mounted on a piezolinear actuator that oscillates at a low frequency of $190 \mathrm{~Hz}$ with a small amplitude of $\sim 100 \mathrm{~nm}$. The choice of frequency has been made in order to avoid any mechanical resonances of the tip. We chose a small amplitude for the same reason. The results are shown in Fig. 6 where we plot the EO signal modulated for tip-crystal separations of $0-1.3 \mu \mathrm{m}$, together with a calculation using the linear wire antenna model. The comparison between the measurement and the calculation clearly shows that the antenna model loses its validity for tip-crystal separations smaller than $0.8 \mu \mathrm{m}$. This increase of the signal as a function of the tip-crystal separation for values smaller than $0.8 \mu \mathrm{m}$ is weaker than predicted by the antenna model. The antenna model therefore is expected to be a valid model for a tip-crystal separation larger than $0.8 \mu \mathrm{m}$.

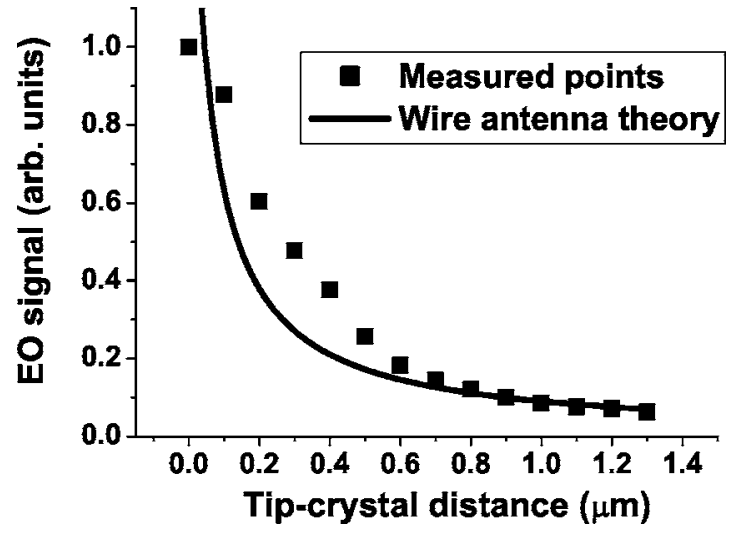

Fig. 6. Measured EO signal as a function of the tip-crystal distance when the tip-crystal separation is modulated. The signal is detected at the tip-crystal modulation frequency. The points represent the measured data, the solid curve is calculated using the antenna model.

\section{DISCUSSION}

Our results clearly point to the shortcomings of the sphere model to describe the near-field of a metal tip. In Section 2, we have presented three models: the sphere model, the cone model, and the antenna model. In Fig. 7, for different lengths of the antenna, the calculated EO signal and also the results of the sphere model are plotted. For small antennas, the two models become identical showing that the sphere model is a special case of the antenna model. The sphere model is unable to explain the slow decay observed when the tip-crystal distance is larger than $2 \mu \mathrm{m}$.

The antenna model, on the other hand, gives an accurate description of the EO signal versus the tip-crystal separation, showing that contributions to the field by the tip shaft are essential to describe our measurements. There are, in fact, several other reasons why treating the tip as a sphere is a bad approximation. First, it was recently shown that metal tips act as low-pass filters for the $\mathrm{THz}$ radiation incident on the metal tip. This can only be explained by assuming that the tip acts like an antenna. It cannot be explained using the sphere model. Second, treating the tip as a sphere strongly underestimates the field enhancement observed underneath the metal tip. This is illustrated by our attempt to measure the $z$ component of the electric field underneath a $16 \mu \mathrm{m}$ diameter gold-coated polystyrene sphere, shown in Fig. 8(a). No measurable signal was obtained. In contrast, in the same Fig. 8(b), we plot the THz electric field strength measured underneath a metal tip with an apex diameter of approximately $1 \mu \mathrm{m}$, showing a strong signal. Third, as mentioned already in Subsection 2.B.1, the calculated symmetry of the $z$ component of the field of a sphere is different from what is measured for a tip. For a sphere, a two-lobed structure is expected. This is illustrated in Fig. 8(c), where we show the signal obtained for a metal sphere of $500 \mu \mathrm{m}$ diameter. We clearly see a dipolelike field with two lobes on each side of the polarization direction, which differs from the one spot seen for the antenna.

Although the frequency filtering properties of our tips have been discussed in an earlier paper, ${ }^{7}$ it is worth mentioning here the results obtained by Chen et al., ${ }^{2}$ who 
measure a change in the near-field spectrum when the tip-sample distance is changed. We do not observe such a change in the spectrum, even when we vary the tipsample distance accurately in the range from 0 to $100 \mu \mathrm{m}$, or when we modulate the tip-sample distance in conjunction with lock-in detection at the modulation frequency. In fact, in our case, the spectral change is determined by the antenna properties of the tip alone and is independent of the tip-sample separation. ${ }^{7}$ We note that Chen's results do not exclude this possibility, as they only plot the difference spectrum obtained from measurements taken at two different heights. Although their spectra can indeed be explained by assuming that the spectrum changes when going from one height to the other, as claimed by the authors, we note that it can equally well be explained by assuming that the spectrum is the same at both heights, but already different from the incident pulse spectrum due to the antenna properties of the tip alone. In the latter case, subtracting the two spectra, which are identical but for their amplitudes, would also produce the spectrum observed by the authors and would also be more in line with our observations.

When the tip-crystal distance is modulated, the measured signal predominantly originates from the apex of the tip. It is tempting to assume that in this case, the signal can be described using a sphere model. We would like to point out, however, that this is incorrect: even though the signal originates from the apex, its strength and reduced bandwidth can only be explained by taking the tip shaft into account as well.

The antenna model does not work very well for a tipcrystal distance smaller than $0.8 \mu \mathrm{m}$. Surprisingly, image dipole effects would make the initial decrease of the signal for increasing tip-crystal separations even steeper. It is therefore not likely that image dipole effects are responsible for the discrepancy between the calculations and the measurements, which show a decrease, which is slower than that of the calculated signal. The experimentally observed slow decay of the signal for distances from 0 to $0.8 \mu \mathrm{m}$ can perhaps also be explained by the finite diameter of the focused probe spot underneath the metal tip. The EO signal then also contains contributions from the tip from locations up to a few micrometers removed from the apex. The summation of the fields of the point

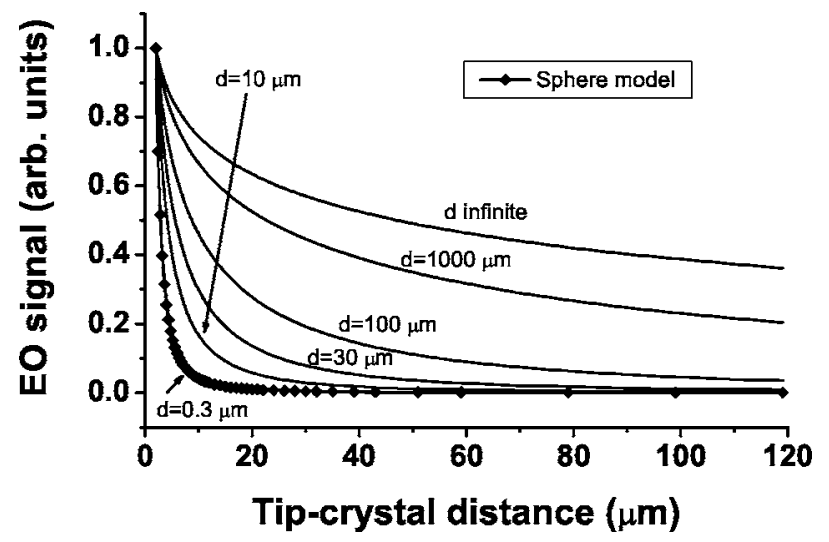

Fig. 7. EO signal calculated for the sphere model and for the antenna model for different lengths $d$ of the antenna with $q$ $=0 \mu \mathrm{m}$ and $p=100 \mu \mathrm{m}$.

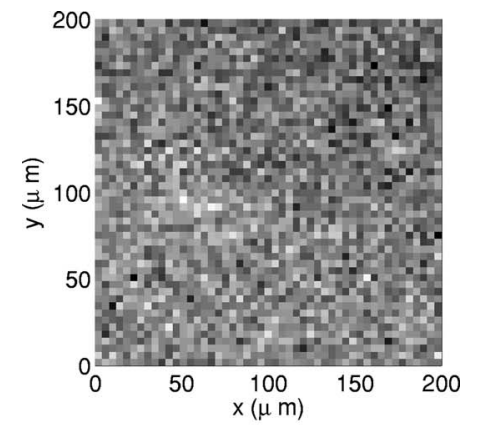

(a)

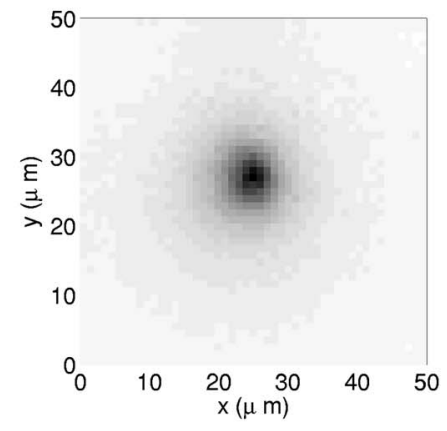

(b)

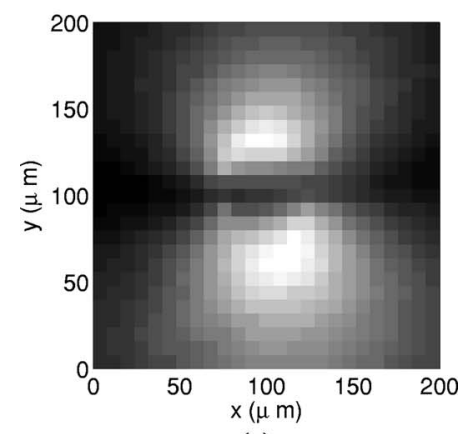

(c)

Fig. 8. THz near-field intensity distribution measured in a plane directly underneath (a) a $16 \mu$ m diameter gold-coated polystyrene sphere, (b) the metal tip, and (c) a $500 \mu \mathrm{m}$ diameter copper sphere.

dipoles along this line then leads to a smearing out of the signal as a function of tip-crystal separation, and thus a slow decay.

\section{CONCLUSIONS}

We have presented calculations and measurements of the THZ electric field in the near field of a metal tip used in the THZ-ANSOM experiment. A comparison with three different models shows that measurements of the electric fields as a function of the tip-crystal separation, can only be explained by treating the tip as a linear wire antenna. Approximating the metal tip by a sphere incorrectly describes the slow decay of the field for increasing tipcrystal distances observed in the experiment. In addition, using the wire antenna model, when we modulate the tipcrystal separation and detect only the modulated signal, we find that the calculated EO signal has the same functional dependence on distance as the field itself, obtained at the surface of the crystal at $z=0$. 


\section{ACKNOWLEDGMENTS}

This work was performed as part of the research program of the Stichting voor Fundamenteel Onderzoek der Materie (FOM), which is financially supported by the Nederlandse Organisatie voor Wetenschappelijk Onderzoek (NWO) and as part of the EU TERANOVA Program (RCN-71835).

A. J. L. Adam's e-mail address is a.j.l.adam@tudelft.nl.

\section{REFERENCES}

1. H.-T. Chen, R. Kersting, and G. C. Cho, "Terahertz imaging nanometer resolution,” Appl. Phys. Lett. 83, 3009-3012 (2003).

2. H. T. Chen, S. Kraatz, G. C. Cho, and R. Kersting, "Identification of a resonant imaging process in aperturless near-field microscopy," Phys. Rev. Lett. 93, 267401 (2004).

3. K. Wang, A. Barkan, and D. M. Mittleman, "Propagation effects in apertureless near-field optical antennas," Appl. Phys. Lett. 84, 305-308 (2004).

4. M. Walther, G. S. Chambers, Z. Liu, M. R. Freeman, and F. A. Hegmann, "Emission and detection of terahertz pulses from a metal-tip antenna," J. Opt. Soc. Am. B 22, 2357-2365 (2005).

5. F. F. Buersgens, H.-T. Chen, and R. Kersting, "Terahertz microscopy of charge carriers in semiconductors," Appl. Phys. Lett. 88, 112115 (2006).

6. N. C. J. van der Valk and P. C. M. Planken, "Electro-optic detection of subwavelength terahertz spot sizes in the near field of a metal tip," Appl. Phys. Lett. 81, 1558-1560 (2002).
7. K. Wang, D. M. Mittleman, N. C. J. van der Valk, and P. C. M. Planken, "Antenna effects in terahertz apertureless near-field optical microscopy," Appl. Phys. Lett. 85, 2715-2717 (2004).

8. P. C. M. Planken and N. C. J. van der Valk, "Spot-size reduction in terahertz apertureless near-field imaging," Opt. Lett. 29, 2306-2308 (2004)

9. J. A. Deibel, N. Berndsen, K. Wang, D. M. Mittleman, N. C. J. van der Valk, and P. C. M. Planken, "Frequencydependent radiation patterns emitted by $\mathrm{THz}$ plasmons on finite length cylindrical metal wires," Opt. Express 14, 8772-8778 (2006).

10. W. Denk and D. W. Pohl, "Near-field optics: microscopy with nanometer-size fields," J. Vac. Sci. Technol. B 9, 510-513 (1991).

11. C. A. Balanis, Antenna Theory, Analysis and Design, 2nd Edition (Wiley, 1997).

12. B. Knoll and F. Keilmann, "Enhanced dielectric contrast in scattering-type scanning near-field optical microscopy," Opt. Commun. 182, 321-328 (2000).

13. J. Van Bladel, "Field singularities at the tip of a cone," Proc. IEEE 71, 901-902 (1983).

14. H. Cory, A. C. Boccara, J. C. Rivoal, and A. Lahrech, "Electric field intensity variation in the vicinity of a perfectly conducting conical probe: application to near-field microscopy," Microwave Opt. Technol. Lett. 18, 120-124 (1998).

15. G. Zhao, R. N. Schouten, N. van der Valk, W. Th. Wenckebach, and P. C. M. Planken, "Design and performance of a $\mathrm{THz}$ emission and detection setup based on a semi-insulating GaAs emitter," Rev. Sci. Instrum. 73 , 1715-1720 (2002). 